\title{
FUNCIONALIDAD FAMILIAR Y DIMENSIONES AFECTIVAS EN ADOLESCENTES ESCOLARIZADOS. CALDAS-COLOMBIA, 2013-2014
}

Consuelo Vélez $A^{1}$, Diana Paola Betancurth L. ${ }^{2}$

\section{Resumen}

Las relaciones familiares representan un aspecto importante del apoyo social en la pre-adolescencia y adolescencia inicial, el objetivo del estudio fue determinar la funcionalidad familiar y su relación con las dimensiones afectivas de los estilos de vida en adolescentes escolarizados residentes en la zona urbana del municipio de Villamaría (Caldas-Colombia). Se diseñó un análisis descriptivo correlacional transversal con 809 estudiantes de instituciones públicas y privadas empleando el APGAR familiar ajustado para escolares colombianos y el instrumento FANTÁSTICO, versión chilena adaptada para la población de estudio. Los resultados mostraron 22,2 por ciento de disfunción familiar entre moderada y severa en los adolescentes participantes, se encontraron diferencias significativas entre la edad, el sexo con el funcionamiento familiar ( $p=0,003$; y $p=0,001$ respectivamente) y una asociación estadísticamente significativa entre el funcionamiento familiar y dimensiones afectivas de los estilos de vida $(p<0,05)$. Los resultados permiten concluir que en la adolescencia el funcionamiento familiar se relaciona directamente con el puntaje de las dimensiones afectivas de los estilos de vida de los adolescentes.

Palabras clave: relaciones familiares, adolescentes, estilo de vida, promoción de la salud APGAR.

${ }^{1}$ Enfermera. Epidemióloga. Doctora en Salud Pública. Docente Departamento Salud Pública. Facultad de Ciencias para la Salud. Universidad de Caldas. Manizales, Grupo Promoción de la Salud y Prevención de la Enfermedad. Universidad de Caldas. E-mail: consuelo.velez@ ucaldas.edu.co. Edificio Miguel Arango SotoCarrera 25 №48-57 (Manizales- Caldas).

${ }^{2}$ Enfermera. Magíster en Enfermería con énfasis en salud familiar. Máster en investigación en Atención Primaria. Candidata a doctor en Salud Pública. Docente Departamento Salud Pública. Facultad de Ciencias para la Salud. Universidad de Caldas. Manizales, Colombia. E-mail: diana.betancurth@ucaldas.edu.co 


\section{FAMILY FUNCTIONALITY AND AFFECTIVE DIMENSIONS IN SCHOOL-GOING ADOLESCENTS. (CALDAS-COLOMBIA) 2013-2014}

Consuelo Vélez A., Diana Paola Betancurth L.

\section{Abstract}

Family relationships signify an important aspect of social support in pre-adolescence and early adolescence; the study objective was to determine the familiar functionality and its relationship with the affective dimensions of the lifestyles in adolescents enrolled in schools living in the urban area of the municipality of Villamaría (Caldas-Colombia). A cross-sectional descriptive correlational analysis with 809 students from public and private institutions was designed, using the familiar APGAR adjusted for Colombian schoolchildren and the instrument FANTÁSTICO, Chilean version adapted for the study of this population. The results showed 22.2 percent of family dysfunction, from moderate to severe in adolescent participants; significant differences were found between age, sex with family functioning ( $p=0.003 ; p=0.001$ respectively) and a statistically significant association between family functioning and emotional dimensions of lifestyle was also found $(p<0.05)$. The results show that in adolescence the family functioning is directly related to the score of the affective dimensions of the lifestyles of adolescents. 


\section{FUNCIONALIDADE FAMILIAR E DIMENSÕES AFETIVAS EM ADOLESCENTES ESCOLARIZADOS. (CALDAS-COLOMBIA) 2013-2014.}

Consuelo Vélez A., Diana Paola Betancurth L.

\section{Resumo}

As relações familiares são um aspecto importante de apoio social na pré-adolescência e início da adolescência, o objetivo do estudo foi determinar a funcionalidade familiar e sua relação com as dimensões afetivas dos estilos de vida em adolescentes escolares que vivem na área urbana do município Villamaría (Caldas-Colômbia). Uma análise de correlação descritiva transversal com 809 estudantes de instituições públicas e privadas usando o APGAR familiar ajustado para escolares colombianos e o instrumento FANTÁSTICO, versão chilena adaptado para a população do estudo foi projetada. Os resultados mostraram 22,2 por cento de disfunção familiar moderada a grave nos adolescentes participantes, encontraram-se diferenças significativas entre a idade, sexo com o funcionamento familiar ( $p=$ 0,003 e $p=0,001$, respectivamente) e uma associação estatisticamente significativa entre funcionamento familiar e dimensões afetivas dos estilos de vida $(p<0,05)$. Os resultados permitem concluir que, durante a adolescência, o funcionamento familiar está diretamente relacionado com a contagem das dimensões afetivas dos estilos de vida dos adolescentes. 


\section{Introducción}

Uno de los principales intereses para la promoción de la salud ha sido el fomento de estilos de vida saludables en las personas, diversos estudios (1-3) han sugerido la importancia de hacerlo en la etapa temprana de la adolescencia $(4-6)^{3}\left(^{*}\right)$ entre la cual se encuentran los/las adolescentes entre los 10 y 15 años, antes de que se adopten comportamientos poco saludables, por lo que el énfasis debe orientarse hacia factores protectores más que a las conductas de riesgo (4). Cuando se habla de factores protectores en salud, significa "hablar de características detectables en un individuo, familia, grupo o comunidad que favorecen el desarrollo humano, el mantenimiento o la recuperación de la salud" (7).

Los estilos de vida saludables de los adolescentes se han concentrado en valorar las condiciones de actividad física, nutrición y conductas problema para la sociedad (drogodependencia, embarazos no deseados, violencia, entre otros) (8), pero hoy se sabe que valorar estos hábitos es aún más complejo, por lo que una visión integral en salud precisa de otras dimensiones de la vida humana que estime también las dimensiones afectividad, la comprensión, la comunicación en las relaciones familiares y de amigos, el tipo de personalidad y la imagen interior (9).
En el desarrollo de los adolescentes se hace especial énfasis en aspectos claves tales como los cambios biopsicosociales en el ciclo de vida, sus características y redes sociales de apoyo, entre los que se encuentran la familia y los amigos (10), dado que es una etapa trascendental del sujeto sobre todo en el ámbito escolar (11). Esta consideración, estimula el interés por estudiar la familia al establecerse como soporte de protección y matriz del desarrollo psicosocial de sus miembros, con el fin de formar la construcción de valores y bases para la autorrealización de los adolescentes (11). La familia constituye la unidad biopsicosocial que permite establecer un sistema saludable donde el adolescente configure hábitos y comportamientos.

Para el caso de esta investigación se asume como familia a todas las personas que un individuo considere como tal y que están emocionalmente conectadas con este, sin importar si están relacionadas biológicamente o viven en la misma casa. Los miembros que la conforman tienen relaciones disimiles entre sí y la familia como unidad tiene a su vez relaciones con sistemas como el educativo y el de salud entre otros (12). Entre tanto, se asume como funcionalidad familiar la capacidad del sistema para enfrentar y superar cada una de las etapas del ciclo vital y las crisis por las que atraviesa (13), Uribe et al. (14) comprenden que el funcionamiento fa-

${ }^{3}$ Clasificaciones actuales diferencian las etapas de desarrollo (pre adolescencia: 9 a 12 años mujeres y varones de 10 a 13 años y adolescencia inicial: 12 a 14 años mujeres y 13 a 15 años varones) adolescencia media: 14 a 16 años mujeres y 15 a 17 años varones, adolescencia tardía: 16 a 18 años mujeres y 17 a 18 años varones, juventud: mujeres y varones entre 18 y 21 años, adolescentes mujeres y varones entre 21 y 24 años (4). 
miliar "se convierte en un aspecto clave durante la adolescencia y la constitución del apoyo social percibido, debido a que es una etapa en donde se enfrenta a intensos cambios de uno o más de sus miembros y por lo tanto cambia su propio comportamiento". En este sentido algunos autores sostienen que es un factor influyente en la conservación de la salud para orientar el fortalecimiento de acciones de todos sus miembros (15, 16).

El estudio se realizó a través de la aplicación de instrumentos de atención a la familia como el APGAR familiar $\left(17^{4}, 18\right)$, al utilizar este, se determina la percepción que tiene el adolescente de cómo está recibiendo el apoyo de su familia en distintas instancias que finalmente hace reportar buena función familiar o mala función familiar. Siendo un instrumento de fácil ejecución, permite evaluar la dinámica familiar y el rol que puede estar jugando en el proceso de la salud como proceso multidimensional (19); evalúa cualitativamente factores como: adaptación, participación, crecimiento, afectividad y resolución, considerados como determinantes en la interrelación familiar. Este instrumento es recomendado por la Guía Técnica Nacional para la Detección Temprana de las Alteraciones del Desarrollo del Joven de 10 a 29 (20), sin embargo el uso que da la guía a esta herramienta es para concentrarse en los factores de riesgo. De otro lado, el instrumento FANTÁSTICO (10) valo- ra diez dimensiones de los hábitos de vida (familiares y amigos, asociatividad y actividad física, nutrición, toxicidad, alcohol, sueño y estrés, tipo de personalidad y actividades, imagen interior, control de la salud y sexualidad y orden), lo que permite una aproximación integral y contrahegemónica a la visión reduccionista de la salud. Para este trabajo se analizó específicamente la dimensión afectiva de los estilos de vida, ya que es la que más estrechamente está relacionada con la funcionalidad familiar y en perspectiva pudiera reflejar las alteraciones de esta última.

En relación a lo anterior, el objetivo de este trabajo fue explorar la relación entre la funcionalidad familiar y las dimensiones afectivas de los estilos de vida en adolescentes escolarizados residentes en la zona urbana del municipio de Villamaría (Caldas-Colombia).

\section{Metodología}

\section{Diseño}

Estudio cuantitativo descriptivo transversal, con análisis correlacional realizado con 809 adolescentes de entre $10 \mathrm{y}$ 15 años, escolarizados en instituciones públicas y privadas del Municipio de Villamaría (Caldas-Colombia) durante el periodo 2013-2014. Como criterio de inclusión se utilizó la edad y la firma del consentimiento informado de los padres y asentimiento de los estudiantes

\footnotetext{
${ }^{4}$ Escala APGAR - Normal: 17-20 puntos Disfunción leve: 16-13 puntos. Disfunción moderada: 12-10 
conseguido en forma individual, además se obtuvo autorización de la alcaldía municipal, secretaria de educación y directivos de las instituciones educativas para la realización de la investigación. Como criterio de exclusión se consideró la posición de aquellos adolescentes quienes a pesar de que sus padres hubieran firmado el consentimiento, no estuvieran de acuerdo.

\section{Instrumentos}

Para hacer la medición del Funcionamiento familiar se utilizó el instrumento APGAR, que según la investigación de Forero et al., fue validado para adolescentes escolarizados en Colombia (20), este instrumento cuenta con buena consistencia interna, dado que los ítems que lo componen se agrupan alrededor de un solo factor con un alfa de Cronbach de 0,793. Para valorar los estilos de vida se utilizó el cuestionario FANTÁSTICO, instrumento adaptado y validado para la población específica en el contexto local sin alterar la valoración del mismo cuyo Alpha de Cronbach fue de 0,80 (21). El cuestionario FANTÁSTICO está compuesto originalmente por 9 dimensiones y 25 preguntas, la propuesta chilena contiene 30 preguntas que se integran en 10 dimensiones; la adaptación que se hizo presenta tres opciones de respuesta con valor numérico de 0 a 2 para cada categoría; las diez dimensiones exploradas fueron: F: familiares y amigos, A: asociatividad y actividad física, $\mathrm{N}$ : nutrición, T: toxicidad A: alcohol, S: sueño y estrés, T: tipo de personalidad y actividades, I: imagen interior, C: control de la salud y sexualidad y finalmente
O: orden $(10,22)$. El instrumento fue auto-diligenciado por los adolescentes, previa presentación de los encuestadores (estudiantes auxiliares de investigación), quienes fueron previamente calibrados por los investigadores, explicaron de forma clara los objetivos, el procedimiento e hicieron el acompañamiento hasta finalizar el proceso. El tiempo para el diligenciamiento del cuestionario FANTÁSTICO por persona fue de aproximadamente 20 minutos y del APGAR familiar otros 20 minutos en promedio.

\section{Análisis estadístico}

Los datos fueron procesados y analizados en el programa SPSS Versión 19.0 licenciado por la Universidad de Caldas, se practicó un análisis univariado y bivariado de la información para mostrar diferencias por sexo y edad en el funcionamiento familiar, además se produjo cruce de variables entre el funcionamiento familiar y las dimensiones afectivas de los estilos de vida relacionados. Todo el estudio se enmarcó dentro de las disposiciones vigentes para la protección de los sujetos humanos que participan en investigación contenidas en la resolución 8430 de 1993 del Ministerio de Salud de Colombia y contempladas en la Declaración de Helsinki clasificando el estudio "con riesgo mínimo”, según el numeral b del Artículo 11 (23).

\section{Resultados}

En relación a las características sociodemográficas el $41,7 \%$ de los participantes fueron mujeres $(n=337)$; el pro- 
medio de edad fue 12,5 años +/- 1,1 años rango (10-15años). Según el grado escolar el $39,9 \%$ se encontraban en grado sexto $30,5 \%$ en séptimo y $29,5 \%$ en octavo.
Frente al funcionamiento familiar se encontró que el $40 \%$ tienen buena función familiar, el 39,8\% tiene disfunción familiar leve y un 7,3\% disfunción familiar severa (Cuadro 1).

Cuadro 1. Funcionamiento familiar de los participantes. $\mathrm{N}=809$

\begin{tabular}{lcc}
\hline \multicolumn{1}{c|}{ Categoría } & Frecuencia & $\%$ \\
\hline Buena función familiar & 324 & 40,0 \\
Disfunción familiar leve & 322 & 39,8 \\
Disfunción familiar moderada & 104 & 12,9 \\
Disfunción familiar severa & 59 & 7,3 \\
Total & $\mathbf{8 0 9}$ & $\mathbf{1 0 0 , 0}$ \\
\hline
\end{tabular}

Fuente: Elaboración propia.

\section{Análisis bivariado}

Se encontraron diferencias estadísticamente significativas $\mathrm{p}<0,05$ al relacionar el género y la edad con el funcionamiento familiar en la población estudiada. Cuadro 2.

Cuadro 2. Variables sociodemográficas vs Disfunción familiar.

\begin{tabular}{|c|c|c|c|c|c|c|c|c|c|}
\hline \multirow[b]{2}{*}{ Variable } & \multirow[b]{2}{*}{ Categorías } & & \multicolumn{4}{|c|}{ Clasificación función familiar } & \multirow[t]{2}{*}{$\mathrm{X}^{2}$} & \multirow[t]{2}{*}{$\mathbf{P}$} & \multirow[t]{2}{*}{$\begin{array}{l}\text { Coeficiente } \\
\text { Contingendia }\end{array}$} \\
\hline & & & $\begin{array}{l}\text { Buena } \\
\text { función } \\
\text { familiar }\end{array}$ & $\begin{array}{r}\text { Disfunción } \\
\text { familiar } \\
\text { leve }\end{array}$ & $\begin{array}{r}\text { Disfunción } \\
\text { familiar } \\
\text { moderada }\end{array}$ & $\begin{array}{r}\text { Disfunción } \\
\text { familiar } \\
\text { severa }\end{array}$ & & & \\
\hline \multirow{4}{*}{ Género } & \multirow{2}{*}{ Femenino } & Frecuencia & 125 & 123 & 56 & 33 & \multirow{4}{*}{14,5} & \multirow{4}{*}{0,003} & \multirow{4}{*}{0,131} \\
\hline & & $\%$ & 37,1 & 36,5 & 16,6 & 9,8 & & & \\
\hline & \multirow{2}{*}{ Masculino } & Frecuencia & 199 & 199 & 48 & 26 & & & \\
\hline & & $\%$ & 42,2 & 42,2 & 10,2 & 5,5 & & & \\
\hline \multirow{4}{*}{ Edad } & \multirow{2}{*}{ 10-12 años } & Frecuencia & 179 & 155 & 43 & 18 & \multirow{4}{*}{15,6} & \multirow{4}{*}{0,001} & \multirow{4}{*}{0,138} \\
\hline & & $\%$ & 45,3 & 39,2 & 10,9 & 4,6 & & & \\
\hline & \multirow{2}{*}{ 13-15 años } & Frecuencia & 145 & 167 & 61 & 41 & & & \\
\hline & & $\%$ & 35,0 & 40,3 & 14,7 & 9,9 & & & \\
\hline
\end{tabular}

Fuente: Elaboración propia. 
De acuerdo a los datos es posible evidenciar una inferencia de sexo en la disfunción familiar, se encontraron ciertas diferencias en la buena función familiar entre hombres $42,2 \%$ y mujeres $37,1 \%$, estas diferencias persisten en la disfunción familiar severa hombres 5,5\% y mujeres $9,8 \%$, lo que permite concluir que el sexo se relaciona directamente con el funcionamiento familiar, en este sentido un mayor porcentaje de hombres tienen una buena función familiar, mientras que las mujeres presentan un mayor porcentaje de disfunción familiar severa.

La edad es otra variable que interfiere en el análisis del funcionamiento familiar, entre los 10-12 años se encontró un $45,3 \%$ de buena función familiar, a diferencia de los rangos de edad entre 1315 años cuyo porcentaje corresponde a un $35,0 \%$, demostrando que en edades menores existe un mayor porcentaje de buena función familiar.

La comunicación familiar presenta una relación directa con la disfunción familiar, en la medida que quienes presentan una disfunción familiar severa, un 52,6\%, enuncian que "nunca" tienen con quien hablar, porcentaje que se reduce a un $5,3 \%$ en quienes tienen una buena función familiar.

En cuanto a la relación entre el funcionamiento familiar y la dimensión afectiva de los estilos de vida en la que se centra el análisis, es posible destacar la expresión de cariño, un $48,9 \%$ de quienes tienen una buena función familiar manifiestan que siempre dan cariño, mientras un $58,3 \%$ de adolescentes que

tienen una disfunción familiar severa nunca dan cariño. Estos hallazgos son similares con la recepción de cariño, un $71,4 \%$ de quienes tienen una disfunción familiar severa enuncian que nunca reciben cariño, porcentaje que se reduce en los adolescentes que tienen buena función familiar representados en un 14,3\% que manifestaron nunca recibir cariño.

Llama la atención la tendencia a la igualdad en los porcentajes de los ítems relacionados con los estados de ánimo y la emocionalidad de los adolescentes, independiente de que los adolescentes tengan una buena función familiar o una disfunción familiar severa, como en el caso de "sin razón me siento molesto/enojado", a lo que un 20,5\% enunciaron "siempre"; en el ítem "sentirse tensos o estresados" en el que $25 \%$ de los adolescentes manifestaron "siempre" sentirse de este modo, esta tendencia se repite en los pensamientos positivos y optimistas, en el cual se destaca la respuesta "nunca" con un $26,3 \%$.

Por otro lado, el buen humor de los adolescentes se relaciona directamente con el funcionamiento familiar, en la medida que quienes tienen una buena función familiar en un 50,3\% siempre se sienten de buen humor, a diferencia de quienes presentan disfunción familiar severa, pues solo un $4 \%$ de ellos dicen sentirse siempre de buen humor.

Estos porcentajes tan distantes no se evidencian en el ítem "me siento contento con mi estudio", en este caso $22 \%$ de los adolescentes que presentan una disfunción familiar severa respondieron "nunca" sentirse contentos con su estudio, un porcentaje que no se aleja del $17,1 \%$ que representa a quienes tienen una buena función familiar. 
Conforme a lo expuesto es posible inferir que el funcionamiento familiar está relacionado con el sexo y la edad, la evidencia muestra que la función familiar tiende a ser buena en los hombres más que en las mujeres y entre los 10 y los 12 años.

De otro lado, se encontró que los escolares con buen funcionamiento familiar tuvieron mejor puntaje en los estilos de vida, y quienes presentan disfunción familiar severa ven afectados sus estilos de vida calificados como estilo de vida bajo y en zona de peligro según el instrumento FANTÁSTICO.

Por último, se identificaron porcentajes similares en quienes tienen una buena función familiar y disfunción familiar severa en ítems relacionadas con los estados de ánimo de los adolescentes, lo que puede corresponder con los cambios propios de las edades de la población sujeto de estudio, cuestiones que en los hallazgos se muestran independientes del funcionamiento familiar.

El Cuadro 3. Muestra las asociaciones encontradas entre el funcionamiento familiar y las variables del estilo de vida en los escolares que tienen que ver con las relaciones afectivas, nótese cómo el funcionamiento familiar presentó asociación estadísticamente significativa con todas las variables analizadas $\mathrm{p}<0,05$.

Cuadro 3. Relación funcionamiento familiar vs dimensión afectiva de los estilos de vida.

\begin{tabular}{|c|c|c|c|c|c|c|c|}
\hline & & \multicolumn{3}{|c|}{ Tengo con quien hablar } & \multirow[t]{2}{*}{$X^{2}$} & \multirow[t]{2}{*}{$P$} & \multirow[t]{2}{*}{$\begin{array}{l}\text { Coeficiente } \\
\text { Contingencia }\end{array}$} \\
\hline & & Nunca & $\begin{array}{l}\text { Algunas } \\
\text { Veces }\end{array}$ & Siempre & & & \\
\hline \multirow{2}{*}{$\begin{array}{l}\text { Buena función } \\
\text { familiar }\end{array}$} & Frecuencia & 1 & 49 & 274 & \multirow{8}{*}{132,08} & \multirow{8}{*}{0,000} & \multirow{8}{*}{0,375} \\
\hline & $\%$ & 5,3 & 20,1 & 50,2 & & & \\
\hline \multirow{2}{*}{$\begin{array}{l}\text { Disfunción } \\
\text { familiar leve }\end{array}$} & Frecuencia & 6 & 120 & 196 & & & \\
\hline & $\%$ & 31,6 & 49,2 & 35,9 & & & \\
\hline \multirow{2}{*}{$\begin{array}{l}\text { Disfunción } \\
\text { familiar moderada }\end{array}$} & Frecuencia & 2 & 46 & 56 & & & \\
\hline & $\%$ & 10,5 & 18,9 & 10,3 & & & \\
\hline \multirow{4}{*}{$\begin{array}{l}\text { Disfunción } \\
\text { familiar severa }\end{array}$} & Frecuencia & 10 & 29 & 20 & & & \\
\hline & $\%$ & 52,6 & 11,9 & 3,7 & & & \\
\hline & & \multicolumn{3}{|c|}{ Yo doy cariño } & & & \\
\hline & & Nunca & $\begin{array}{l}\text { Algunas } \\
\text { Veces }\end{array}$ & Siempre & \multirow{9}{*}{67,99} & \multirow{9}{*}{0,000} & \multirow{9}{*}{0,278} \\
\hline \multirow{2}{*}{$\begin{array}{l}\text { Buena función } \\
\text { familiar }\end{array}$} & Frecuencia & 1 & 146 & 177 & & & \\
\hline & $\%$ & 8,3 & 33,7 & 48,6 & & & \\
\hline \multirow{2}{*}{$\begin{array}{l}\text { Disfunción } \\
\text { familiar leve }\end{array}$} & Frecuencia & 4 & 189 & 129 & & & \\
\hline & $\%$ & 33,3 & 43,6 & 35,4 & & & \\
\hline \multirow{2}{*}{$\begin{array}{l}\text { Disfunción } \\
\text { familiar moderada }\end{array}$} & Frecuencia & 0 & 68 & 36 & & & \\
\hline & $\%$ & 0 & 15,7 & 9,9 & & & \\
\hline \multirow{2}{*}{$\begin{array}{l}\text { Disfunción } \\
\text { familiar severa }\end{array}$} & Frecuencia & 7 & 30 & 22 & & & \\
\hline & $\%$ & 58,3 & 6,9 & 6,0 & & & \\
\hline
\end{tabular}


Continuación Cuadro 3. Relación funcionamiento familiar vs dimensión afectiva de los estilos de vida.

\begin{tabular}{|c|c|c|c|c|c|c|c|}
\hline & & \multicolumn{3}{|c|}{ Yo recibo cariño } & \multirow[t]{2}{*}{$\mathrm{X}^{2}$} & \multirow[t]{2}{*}{$P$} & \multirow[t]{2}{*}{$\begin{array}{l}\text { Coeficiente } \\
\text { Contingencia }\end{array}$} \\
\hline & & Nunca & $\begin{array}{l}\text { Algunas } \\
\text { Veces }\end{array}$ & Siempre & & & \\
\hline \multirow{2}{*}{$\begin{array}{l}\text { Buena función } \\
\text { familiar }\end{array}$} & Frecuencia & 1 & 34 & 289 & \multirow{8}{*}{151,28} & \multirow{8}{*}{0,000} & \multirow{8}{*}{0,397} \\
\hline & $\%$ & 14,3 & & & & & \\
\hline \multirow{2}{*}{$\begin{array}{l}\text { Disfunción } \\
\text { familiar leve }\end{array}$} & Frecuencia & 1 & 99 & 222 & & & \\
\hline & $\%$ & 14,3 & & & & & \\
\hline \multirow{2}{*}{$\begin{array}{l}\text { Disfunción } \\
\text { familiar moderada }\end{array}$} & Frecuencia & 0 & 47 & 57 & & & \\
\hline & $\%$ & 0 & & & & & \\
\hline \multirow{2}{*}{$\begin{array}{l}\text { Disfunción } \\
\text { familiar severa }\end{array}$} & Frecuencia & 5 & 37 & 17 & & & \\
\hline & $\%$ & 71,4 & & & & & \\
\hline
\end{tabular}

Sin razón me siento molesto y/o enojado

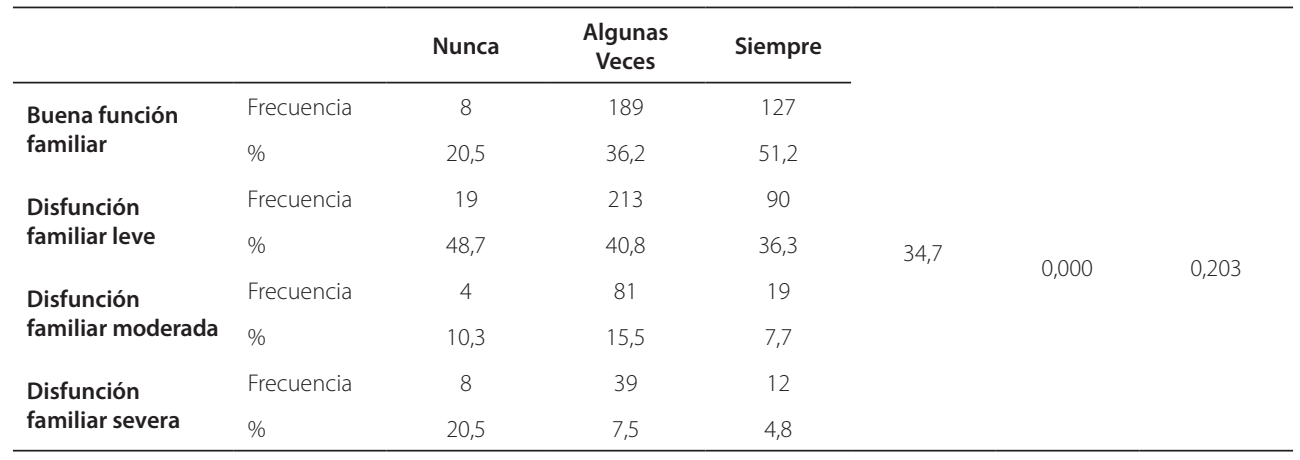

Me siento de buen humor alegre y activo

\begin{tabular}{|c|c|c|c|c|c|c|c|}
\hline & & Nunca & $\begin{array}{l}\text { Algunas } \\
\text { Veces }\end{array}$ & Siempre & & & \\
\hline \multirow{2}{*}{$\begin{array}{l}\text { Buena función } \\
\text { familiar }\end{array}$} & Frecuencia & 3 & 120 & 201 & & \multirow{8}{*}{0,000} & \multirow{8}{*}{0,272} \\
\hline & $\%$ & 20 & 30,5 & 50,3 & & & \\
\hline \multirow{2}{*}{$\begin{array}{l}\text { Disfunción } \\
\text { familiar leve }\end{array}$} & Frecuencia & 5 & 169 & 148 & & & \\
\hline & $\%$ & 33,3 & 42,9 & 37 & & & \\
\hline \multirow{2}{*}{$\begin{array}{l}\text { Disfunción } \\
\text { familiar moderada }\end{array}$} & Frecuencia & 1 & 68 & 35 & \multirow{4}{*}{64,80} & & \\
\hline & $\%$ & 6,7 & 17,3 & 8,8 & & & \\
\hline \multirow{2}{*}{$\begin{array}{l}\text { Disfunción } \\
\text { familiar severa }\end{array}$} & Frecuencia & 6 & 37 & 16 & & & \\
\hline & $\%$ & 40 & 9,4 & 4 & & & \\
\hline
\end{tabular}

Me siento contento con mi estudio

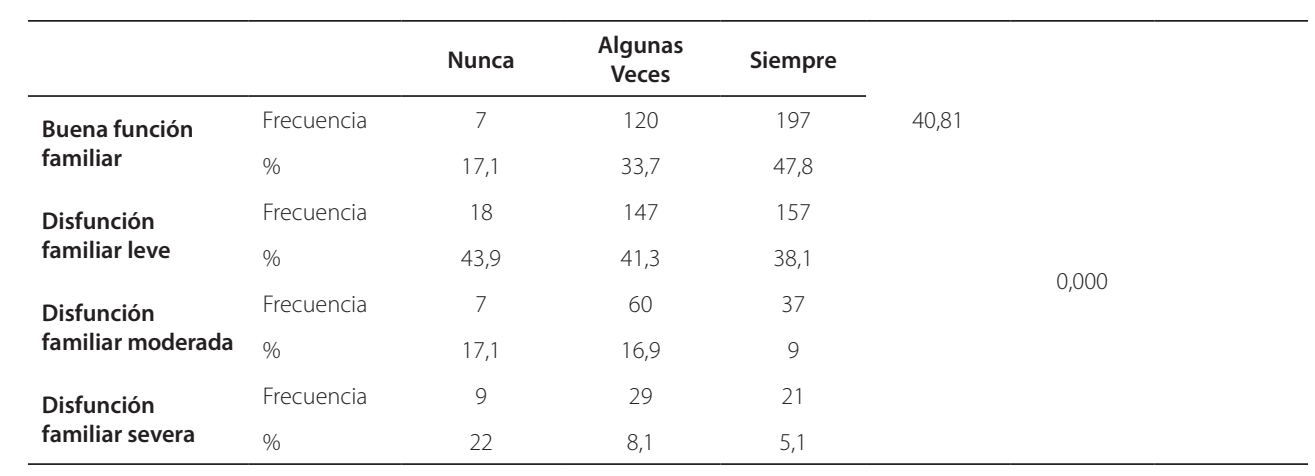


Continuación Cuadro 3. Relación funcionamiento familiar vs dimensión afectiva de los estilos de vida.

\begin{tabular}{|c|c|c|c|c|c|c|c|}
\hline & & \multicolumn{3}{|c|}{$\begin{array}{l}\text { Tengo pensamientos positivos y } \\
\text { optimistas }\end{array}$} & \multirow[t]{2}{*}{$\mathrm{X}^{2}$} & \multirow[t]{2}{*}{$P$} & \multirow[t]{2}{*}{$\begin{array}{l}\text { Coeficiente } \\
\text { Contingencia }\end{array}$} \\
\hline & & Nunca & $\begin{array}{l}\text { Algunas } \\
\text { Veces }\end{array}$ & Siempre & & & \\
\hline \multirow{2}{*}{$\begin{array}{l}\text { Buena función } \\
\text { familiar }\end{array}$} & Frecuencia & 5 & 79 & 240 & \multirow{6}{*}{80,7} & & \\
\hline & $\%$ & 26,3 & 25,1 & 50,5 & & & \\
\hline \multirow{2}{*}{$\begin{array}{l}\text { Disfunción } \\
\text { familiar leve }\end{array}$} & Frecuencia & 8 & 136 & 178 & & & \\
\hline & $\%$ & 42,1 & 43,2 & 37,5 & & \multirow{5}{*}{0,000} & \multirow{5}{*}{0,301} \\
\hline \multirow{2}{*}{$\begin{array}{l}\text { Disfunción } \\
\text { familiar moderada }\end{array}$} & Frecuencia & 1 & 67 & 36 & & & \\
\hline & $\%$ & 5,3 & 21,3 & 7,6 & & & \\
\hline \multirow{2}{*}{$\begin{array}{l}\text { Disfunción } \\
\text { familiar severa }\end{array}$} & Frecuencia & 5 & 33 & 21 & & & \\
\hline & $\%$ & 26,3 & 10,5 & 4,4 & & & \\
\hline
\end{tabular}

Me siento tenso y estresado

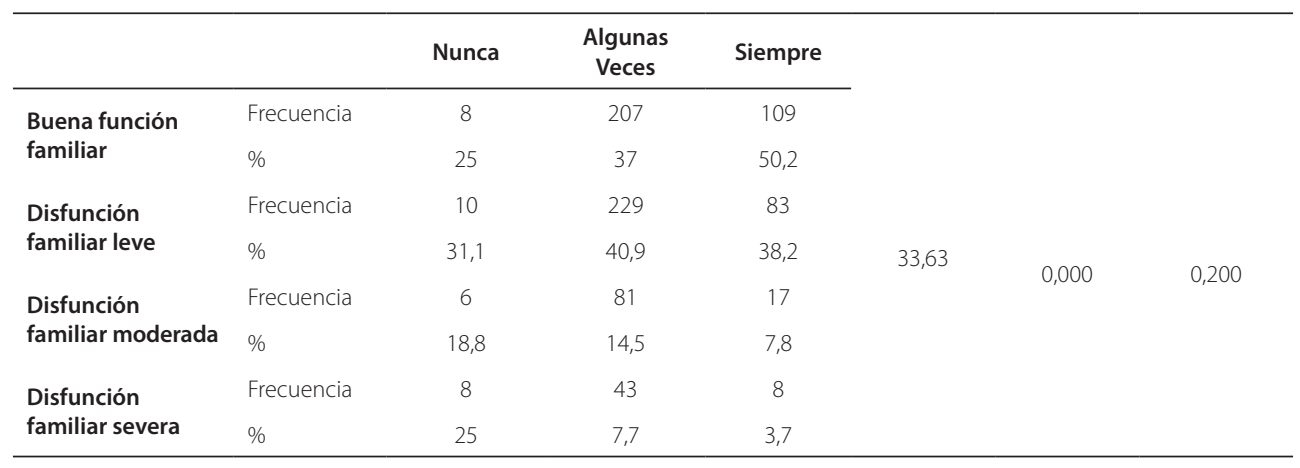

Me siento deprimido o triste

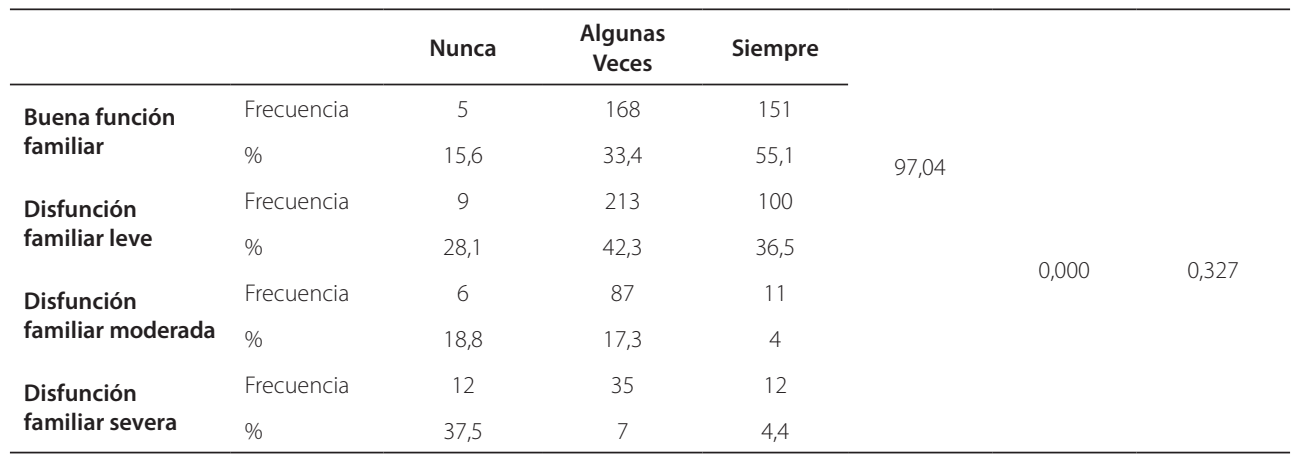

Fuente: Elaboración propia.

\section{Discusión}

El principal objetivo de la presente investigación fue determinar la funcio- nalidad familiar y su relación con las dimensiones afectivas de los estilos de vida de adolescentes escolarizados entre 10 y 15 años de edad de la zona urbana 
tados arrojados indican la presencia de una disfunción familiar entre moderada y severa en el $22,2 \%$ de la población sujeto de estudio, además de asociaciones significativas diferentes para hombres y mujeres entre los subsistemas familiares, lo que podría influir de la misma manera en los estilos de vida saludables de los adolescentes.

De acuerdo a lo anterior, es importante trascender los lineamientos de un modelo biomédico dominante como el de la guía técnica nacional colombiana para atención de adolescentes y jóvenes, la cual demarca esta etapa como vulnerable a factores de riesgo intrínsecos y extrínsecos, lo que convierte a esta población en una prioridad en la atención en salud que se enfoca solamente en problemáticas como: delitos sexuales, suicidios, homicidios, muertes accidentales, explotación sexual, limitaciones de carácter cognitivo, sensorial o motor, la fecundidad en adolescentes, muertes maternas, Virus de Inmuno deficiencia Humana (VIH), Síndrome de Inmunodeficiencia Adquirido (SIDA), alcohol y trastornos del comportamiento alimentario, sin tener en cuenta precisamente esas interacciones y relaciones afectivas entre los miembros de la familia, y otras variables como el sexo y la edad que son consideradas en esta investigación y cuyos resultados soportan su importancia para potenciar factores protectores con acciones conjuntas de promoción para la salud (20).

En este sentido, es necesario partir de la familia y su función, a pesar de que esta dependa de múltiples variables como el sexo, la edad y la estructura del hogar, as- pectos que han cambiado como parte de un proceso histórico. Su adecuado funcionamiento debe estar orientado a satisfacer las necesidades afectivas de cada uno de los miembros para promover el desarrollo integral del entorno familiar, principalmente durante la adolescencia inicial donde es preciso centrarse en una dinámica familiar positiva, basada en la comunicación como una de las conductas saludables con la que los familiares interactúan y comparten mensajes de comprensión, respeto y apoyo emocional (24).

Expresar afecto y brindar protección es una función primordial en la familia, en esta unidad psicosocial, los vínculos adecuados entre padres e hijos se susten$\tan$ en relaciones en que se expresa cariño, y fuertes los lazos fraternales entre sus miembros, tanto padres e hijos como en la pareja misma. Las conexiones de parentesco son más sólidas y permanentes en tanto y en cuanto se basen en afectos positivos (25). Esta interrelación permite que los adolescentes fortalezcan su esfera emocional, situación que ha sido corroborada con los resultados del presente estudio.

Dichos lazos se relacionan con las dimensiones afectivas de los estilos de vida, los cuales son adquiridos en los grupos sociales, la familia, los amigos, la escuela, entre otros, los que forman patrones conductuales sanos que los adolescentes pueden usar de manera consciente o inconsciente (10), y que tienen que ver con factores protectores como dar cariño, estar de buen humor, estar contento en el estudio y tener pensamientos positivos en los adolescentes, 
lo cual se hizo evidente al examinar los resultados y encontrar una asociación estadísticamente significativa entre el funcionamiento familiar y las variables de la dimensión afectiva. En éste mismo sentido el trabajo desarrollado por Alonso concluye que la funcionalidad familiar es muy importante ya que afecta significativamente las conductas de los adolescentes (26).

En relación a lo mencionado, diferentes autores y estudios $(24,27,28)$ demuestran que la función afectiva familiar es una excelente forma de proteger la salud familiar integral, pues cuando los padres o adultos ejercen de manera positiva la disciplina consistente y el apoyo emocional, los adolescentes tienen menos problemas y se alejan de conductas riesgosas que se caracterizan por amenazar su desarrollo personal, su salud y sus roles sociales.

El resultado final de esta investigación refleja la relación existente entre el funcionamiento familiar y la calificación del estilo de vida de los adolescentes, reafirmando la importancia de las dimensiones emocionales y afectivas presentes en un adecuado funcionamiento familiar, la mejora en estas dimensiones permite consolidar a la familia como fuente de apego positivo durante esta etapa, definiéndose como un contexto cohesivo que mitiga los factores y las situaciones de riesgo para esta población (29) que atraviesa complejos cambios físicos, psicológicos, cognoscitivos, emocionales y sociales, propios de esta etapa y por tanto deben ser comprendidos y sorteados en el entorno familiar (10).
Este estudio evidencia la necesidad de abordar las dimensiones humanas, emocionales y afectivas en las diferentes estrategias de promoción para la salud en adolescentes, pues estas dimensiones a menudo son asumidas por profesionales de las ciencias sociales, como psicólogos o trabajadores sociales, lo que deja ver un modelo de salud hegemónico y fragmentado que se concentra únicamente en la enfermedad excluyendo aspectos familiares, individuales y sociales, que hacen parte de una visión de salud como vida (30) y por ende deben ser prioridad para los salubristas.

\section{Conclusiones}

Existe un vacío en la promoción para la salud en cuanto al análisis de las dimensiones afectivas y emocionales de los adolescentes, la presente investigación responde a este cuestionamiento y permite identificar la afectividad como una de las dimensiones más importantes en el desarrollo de estrategias de promoción de la salud en la población sujeto de estudio, al evidenciar la relación entre el adecuado funcionamiento familiar y la calificación de los estilos de vida en los adolescentes.

Los hallazgos de este estudio también amplían la concepción de los estilos de vida más allá de sus dimensiones corporales relacionadas principalmente con la nutrición y la actividad física, para tener en cuenta aquellas cuestiones emocionales y afectivas presentes en el ámbito familiar que contribuyen al desarrollo integral de los adolescentes, su comprensión y soporte como parte de un buen funcionamiento familiar. 
En este sentido, desde la promoción para la salud es necesario hacer una evaluación integral incluyendo la función familiar, teniendo en cuenta el enfoque de género y la pluralidad de las dinámicas familiares, en esta evaluación corresponde analizar sus múltiples dimensiones (biológica, psicológica, social y cultural), ya que en esta etapa se consolidan los estilos de vida que serán determinantes para las etapas posteriores, además de implementar diferentes estrategias que involucren a la familia y la conciban no como un ámbito privado, sino como un sistema que se relaciona con otros $y$ en esta medida promuevan espacios de integración, recreación y aprendizaje útiles para el mejoramiento de la salud familiar.

\section{Referencias}

1. Palenzuela SM. Hábitos y conductas relacionados con la salud de los escolares de $6^{\circ}$ de primaria de la provincia de Córdoba [Tesis doctoral]. Córdoba: Servicio de Publicaciones de la Universidad de Córdoba; 2010. Consultado el Fecha de consulta y 11 de noviembre de 2016. Disponible en: http://helvia.uco.es/xmlui/bitstream/ handle/10396/3659/9788469363959.pdf?sequence

2. Departamento para la Prosperidad Social, Ministerio de la Protección Social, INS, ICBF. Encuesta Nacional de la Situación Nutricional en Colombia. ENSIN 2010. Consultado el 12 de enero 2015. Disponible en: http://www.icbf.gov.co/icbf/ directorio/portel/libreria/pdf/Resumenfi. pdf

3. Turconi G, Guarcello M, Maccarini L, Cignoli F, Setti S, Bazzano R, Roggi C. Eating habits and behaviors, physical activity, nutritional and food safety knowledge and beliefs in an adolescent Italian population. J Am Coll Nutr. 2008. Consultado el 12 de diciembre de 2016. 27(1): 31-43.
Disponible en: ttps://www.ncbi.nlm.nih. gov/pubmed/18460479

4. Breinbauer $C$, Maddaleno $M$. Jóvenes: opciones y cambios: promoción de conductas saludables en los adolescentes. Publicación Científica y Técnica, 594. Washington, D.C: OPS; 2008. Consultado el 11 de diciembre de 2015 Fecha de consulta y citación 2015/12/11. Disponible en: http://publications.paho.org/spanish/ PC+594+cap_7.pdf

5. Ministerio de Salud. Norma técnica para la detección temprana de alteraciones del desarrollo del joven de 10 a 29 años. Resolución 412 de 2000. Consultado el 11 de octubre de. Disponible en. https://www.minsalud.gov.co/sites/rid/Lists/ BibliotecaDigital/RIDE/VS/PP/7Deteccion $\% 20$ temprana $\% 20$ alteraciones $\% 20$ joven.pdf

6. Betancurth DP, Vélez C. La adolescencia: un reto para los profesionales de la salud. Revista Cultura del Cuidado. 2012; 9(2). Disponible en:http://repositorio.uni- 
librepereira.edu.co:8080/pereira/handle/123456789/170

7. Páramo MA. Factores de riesgo y factores de protección en la adolescencia: análisis de contenido a través de grupos de discusión. Terapia Psicológica. 2011. Consultado el 12 de junio de 2015. 29(1): 85-95. Disponible en: http://www.redalyc. org/articulo.oa?id=78518428010

8. Barra E, Cancino V, Lagos G, Leal P, San Martín JS. Factores psicosociales y problemas de salud reportados por adolescentes. Psicología y salud. 2005. Consultado el 13 de diciembre de 2015. 15(2): 231-239. Disponible en. http://revistas.uv.mx/index.php/psicysalud/article/ view/807/1463

9. Wilson DM, Ciliska D. Life-style assessment: Development and use of the FANTASTIC checklist. Can Fam Physician. 1984; 30: 1527-32.

10. Orcasita L, Uribe A. La importancia del apoyo social en el bienestar de los adolescentes. Psychologia: avances de la disciplina. 2010. Consultado el 23 de noviembre de 2015. 4(2): 69-82. Disponible en: http://132.248.9.34/hevila/PsychologiaAvancesdeladisciplina/2010/vol4/ no2/6.pdf

11. Gutiérrez- P, Camacho N, MartínezML. Autoestima, funcionalidad familiar y rendimiento escolar en adolescentes. Atención Primaria. 2007. Consultado el 23 de enero de 2016. 39(11): 597-601. Disponible en: http://www.elsevier.es/ es-revista-atencion-primaria-27-linkresolver-autoestima-funcionalidad-familiar-rendimiento-escolar-13112196

12. Doccal, M., Clavijo, A., Barajas, G. y Cortés, L. Concepciones sobre familia en adolescentes escolarizados en instituciones educativas distritales en Bogotá, Colombia. Cultura Educación y Sociedad. 2016. Consultado el 23 de diciembre 2014. 7(1), 23-38. Disponible en: file:///Users/ofinvestigaciones/Downloads/1070-5883-1-PB.pdf
12. Higuita LF, Cardona JA. Percepción de funcionalidad familiar en adolescentes escolarizados en instituciones educativas públicas de Medellín (Colombia), 2014. Rev. CES Psicología. 2016 Consultado el 24 de febrero de 2016. 9(2):167-179. Disponible en: http://revistas.ces.edu.co/index.php/psicologia/article/view/3600/2660

13. Uribe A, Orcasita L, Aguillón E. Bullying, redes de apoyo social y funcionamiento familiar en adolescentes de una institución educativa de Santander, Colombia. Psychologia: avances de la disciplina. 2012. Consultado el 21 de febrero de 2015. 6(2): 83-99. Disponible en: http://www.scielo.org.co/pdf/psych/v6n2/ v6n2a08.pdf

15. Hidalgo CG. Evaluación del funcionamiento familiar en familias con y sin riesgo psicosocial. Psykhe. 1999. Consultado el 12 de diciembre de 2015. 8(2): 85-101. Disponible en: www.psykhe.cl/index.php/ psykhe/article/download/135/133

16. Rangel JL, Valerio L, Patiño J, García M. Funcionalidad familiar en la adolescente embarazada. Rev Fac Med UNAM. 2004; 47(1): 24-27.

17. Suarez MA, Alcalá M. APGAR familiar: una herramienta para detectar disfunción familiar. Rev. Méd. La Paz. 20(1)1. Consultado el 11 de diciembre de 2016. Disponible en: http://www.scielo. org.bo/scielo.php?script=sci_arttext\&pi$d=S 1726-89582014000100010$

18. Forero LM, Avendaño MC, Duarte ZJ, Campo A. Consistencia interna y análisis de factores de la escala APGAR para evaluar el funcionamiento familiar en estudiantes de básica secundaria. Revista Colombiana de Psiquiatría. 2006. Consultado el 12 de diciembre de 2015; 35(1): 23-29. Disponible en: http://www.redalyc. org/articulo.oa? id=80635103

19. Breilh J. Epidemiología crítica. Ciencia emancipadora e interculturalidad. Buenos Aires: Lugar Editorial; 2003. Consultado el 12 de noviembre de 2015. Disponible 
en:http://www.sld.cu/galerias/pdf/sitios/revsalud/18_epidemiologiacritica.pdf

20. Ministerio de la Protección Social. Norma técnica para la detección temprana de las alteraciones del desarrollo del joven de 10 a 29 años. 2000. Consultado el 12 de enero de 2015. Disponible en: http://207.58.191.15:8180/xmlui/bitstream/handle/123456789/46/Legal_38. pdf?sequence $=1$

21. Betancurth DP, Vélez $C$, Jurado $L$. Validación de contenido y adaptación del cuestionario Fantástico por técnica Delphi. Salud Uninorte. 2015. Consultado el 23 de diciembre 2014; 2: 214-227. Disponible en: http://rcientificas.uninorte. edu.co/index.php/salud/article/viewArticle/5583/8016

22. Lange I, Vio F. Guía para universidades saludables y otras instituciones de educación superior. Santiago de Chile: Andros Impresores. Consultado el 12 de enero de 2016. Disponible en: http://www.eligevivirsano.cl/wp-content/ uploads/2012/01/Guia-Universidades-Saludables_INTAOPS.pdf

23. Ministerio de Salud. Resolución No. 008430 de 1993, por la cual se establecen las normas científicas, técnicas y administrativas para la investigación en salud. Consultado el 13 de noviembre de 2015 2015/11/13. Disponible en: http:// comitedeetica.clinicalili.org/docs/RESL8430.pdf

24. Pi AM, Cobián A. Componentes de la función afectiva familiar: una nueva visión de sus dimensiones e interrelaciones. MEDISAN. 2009; 13(6). Disponible en: http://scielo.sld.cu/scielo.php?script=sci arttext\&pid=S1029-30192009000600016

25. Pi AM, Cobián A. Desarrollo del clima familiar afectivo y su impacto en el bien- estar subjetivo de la familia. Multimed 2015. Consultado el 19 de diciembre de 2015;19(2):33-44. Disponible en: http:// www.revmultimed.sld.cu/index.php/mtm/ article/view/239/573

26. Alonso MM, Yañe Á, Armendáriz NA, funcionalidad familiar y consumo de alcohol en adolescentes de secundaria. Salud y drogas 2017. Consultado el 17 de febrero 2017; 17(1):87-96. Disponible en: http://www.redalyc.org/articulo. oa?id=83949782009. .

27. Carrillo S, Ripoll-Núñez K, Cabrera VE, Bastidas H. Relaciones familiares, calidad de vida y competencia social en adolescentes y jóvenes colombianos. Summa Psicológica UST. 2009. Consultado el 23 de octubre de 2015; 6(2): 3-18. Disponible en:http://132.248.9.34/hevila/SummapsicologicaUST/2009/vol.6/ no2/1.pdf

28. Perdomo AY, Ramírez CA, Galán EF. Asociación de la funcionalidad familiar entre padres e hijos adolescentes en Neiva. Enferm. 2015. Consultado el 22 de enero de 2015; 33(1): 94-103. Disponible en: http://www.scielo.org. co/scielo.php?script=sci_abstract\&pi$\mathrm{d}=$ S0121-45002015000100011

29. Luna AC. Funcionamiento familiar, conflictos con los padres y satisfacción con la vida de familia en adolescentes bachilleres. Acta Colombiana de Psicología. Consultado el 23 de noviembre de 2015; 5(1): 77-85. Disponible en: http://www.redalyc. org/articulo. oa?id=79824560008

30. Granda E. La salud pública y las metáforas sobre la vida. Revista Facultad Nacional de Salud Pública. 2000. Consultado el 7 de diciembre de 2015; 18(2): 83100. Disponible en: http://www.redalyc. org/pdf/120/12018207.pdf 